\title{
Tutorial de Pubmed
}

\section{Página de resultados}

Una vez realizada la búsqueda (tanto en el modo básico como en e avanzado), en esta página se muestra una lista de las citas obtenidas de acuerdo a la estrategia de búsqueda realizada. Por ejemplo, si en el modo básico de búsqueda ingresamos la palabra clave neoplasm en el casillero de texto como lo muestra la imagen, (recuerde que por defecto la base en donde se realizará la búsqueda es en el Medline pero podríamos elegir otras) y se limita la recuperación de citas a los últimos dos años y la cantidad de citas a 10 por página, se vería de la siguiente forma:

$\begin{aligned} & \text { Search } \\ & \\ & \text { neoplasm }\end{aligned}$
Number of documents to display per page $10 \quad \checkmark$
Entrez Date Limit 2 Years $\nabla$

Luego de oprimir el botón "Search", y que el motor de búsqueda de PubMed procese el pedido nos lleva a la Página de resultados o "The Document Summary Page" la cual se vería de la siguiente forma:

NCBI PubMed PubMed QUERY PubMed ?
(past 2 Years only)

$\begin{array}{lll}\text { Details } & \text { Search Clear } 1 \\ \text { Docs Per Page: } & 10 \quad \text { Entrez Date Limit: } 2 \text { Years - }\end{array}$

citations 1-10 displayed (out of 101160 found) page 1 of 10116

Display Abstract report $\nabla$ for the article selected (default all)

order documents on this page through Loansome Doc

\section{$\square$ Chedid A. et al [See Related Articles]}

Morphology and Other Prognostic Factors of Hepatocellular Carcinoma. Arch Pathol Lab Med. 1999 Jun;123(6):524-528.

[Record as supplied by publisher] PMID: 10383806.

\section{$\square$ Matsumoto T. et al [See Related Articles]}

Leiomyoma with atypical cells (atypical leiomyoma) in the larynx. Histopathology. 1999 Jun;34(6):532-536. [Record as supplied by publisher] PMID: 10383698.

En dicha página en primera instancia aparece el casillero de texto donde ingresamos el término de búsqueda o keyword (1) con las limitaciones que se pudieron haber efectuado (tanto la de tiempo como el número de citas por página). Inmediatamente por debajo nos informa la cantidad de citas encontradas (2) y la forma en la que están siendo presentadas (cantidad de citas por página y cantidad de páginas)

Luego se nos presentan dos botones (3), el de "Display" y un botón de menú extensible, el "Viewing Formats", este último nos da la posibilidad de elegir el tipo de formato en el que se presentarán las citas obtenidas en la búsqueda (este punto se amplía más adelante).

Por debajo encontramos (4) las citas propiamente dichas, mostradas según el formato de presentación de la página de resultados. Siguiendo el ejemplo tomaremos la primera de las citas para describir las posibles formas de presentación.

\section{Formatos de presentación de las citas}

\section{Formato mínimo:}

Por defecto las citas en la página de resultados se presentan en el formato mínimo, tomando la primera cita como ejemplo observemos sus componentes:

\section{Chedid A. et al 1 [See Related Articles]}

2 Morphology and Other Prognostic Factors of Hepatocellular Carcinoma.

Arch Pathol Lab Med. 1999 Jun;123(6):524-528.
[Record as supplied by publisher]

4 PMID: 10383806.

1) Primer autor: el primer autor en sí es un enlace a otro formato de presentación (por defecto es el "abstract format" o resumen).

2) Título: en primer término el título del artículo en cuestión. A continuación del punto final del título los reportes podrían contener anotaciones adicionales, tales como: [MEDLINE record in process] : cita del PreMEDLINE, artículos que ingresan a la base de datos pero que aún no tiene adjudicados los términos MeSH correspondientes.

Lenguaje, las artículos que no fueron escritos originalmente en inglés (aunque si poseen abstract, mostrado en inglés) poseen una nota que hace referencia al lenguaje. Por ej.: French.

El tipo de publicación, en nuestro ejemplo vemos que se trata de un "trial clínico randomizado".

3) Fuente bibliográfica: en el formato del Medline, se presenta la fuente de donde se publicó el artículo al que la cita hace referencia, con la fecha de publicación, el volumen y el número de página.

4) Número identificatorio de MEDLINE y PubMed: el PMID es el número identificatorio de la cita en la base del PubMed y el UI es el número con el que se identifica al artículo en el Medline propiamente dicho.

En este formato mínimo cada una de las citas tiene un casillero de selección en el margen izquierdo del primer autor, el cual sirve para marcar los artículos que se quieren ver en otro tipo de formato o para ver el abstract en el formato por defecto (abstract report).

Es importante saber que si no se selecciona ninguno por regla implícita se mostrarán todas las citas de la página al oprimir el botón "Display" (y en el formato seleccionado).

Si se desea ver solamente una de las citas se debe seguir el link ofrecido en el hipertexto del primer autor del artículo. De la misma manera se pueden ver los artículos relacionados (siempre de cada cita) en el hipertexto de "See Related Articles" (se amplía más adelante).

Luego de haber seleccionado las citas que se desean ver en otro formato (o todas por defecto) debemos elegir el tipo de formato de presentación del menú desplegable del "Viewing Format" como se ve en la imagen a la derecha.

Generalmente se debe optar por el "Abstract report" ya que este nos muestra los datos ya vistos en el formato mínimo de la página de resultados con algunas modificaciones y obviamente, si la cita lo tuvie-

\begin{tabular}{|l|}
\hline Abstract report \\
\hline Citation report \\
Abstract report \\
MEDLINE report \\
ASN 1 report \\
Related articles \\
protein links \\
nucleotide links \\
structure links \\
genome links \\
\hline
\end{tabular}


ra, el abstract del artículo; recuerde que NUNCA encontrará artículos completos (full-text) en el Medline, aunque ya veremos como PubMed nos dirije en algunos artículos al texto completo en su versión electrónica en la Web. Describiremos a continuación alguno de los formatos de presentación:

\section{Abstract report:}

Una vez seleccionadas las citas a ver, y habiendo elegido el formato de presentación "Abstract report" (recuerde que por defecto ya está seleccionado) se debe oprimir el botón "Display", o siguiendo el enlace ofrecido en el primer autor, pasamos a la página con el formato del "Abstract report". La siguiente imagen lo representa, veamos sus componentes:

NCBI PubMed PubMed QUERY PubMed ?

Other Formats: Citation MEDLINE 1

Links: Related Articles

$\square$ Order this document

JAMA 1999 Jun 16;281(23):2189-97

2

The effect of raloxifene on risk of breast cancer in postmenopausal women: results from the MORE randomized trial. Multiple Outcomes of Raloxifene Evaluation.

Cummings SR, Eckert S, Krueger KA, Grady D, Powles TJ, Cauley JA, Norton L, Nickelsen T, Bjarnason NH, Morrow M, Lippman ME, Black D, Glusman JE, Costa A, Jordan VC.

Department of Medicine, University of California, San Francisco, USA. scummings@psg.ucsf.edu

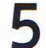

CONTEXT: Raloxifene hydrochloride is a selective estrogen receptor modulator that has antiestrogenic effects on breast and endometrial tissue and estrogenic effects on bone, lipid metabolism, and blood clotting. OBJECTIVE: To determine whether women taking raloxifene have a lower risk of invasive breast cancer. DESIGN AND SETTING: The Multiple Outcomes of Raloxifene Evaluation (MORE), a multicenter, randomized, double-blind trial, in which women taking raloxifene or placebo were followed up for a median of 40 months (SD, 3 years), from 1994 through 1998, at 180 clinical centers composed of community settings and medical practices in 25 countries, mainly in the United States and Europe. PARTICIPANTS: A total of 7705 postmenopausal women, younger than 81 (mean age, 66.5 ) years, with osteoporosis, defined by the presence of vertebral fractures or a femoral neck or spine T-score of at least 2.5 SDs below the mean for young healthy women. Almost all participants $(96 \%)$ were white. Women who had a history of breast cancer or who were taking estrogen were excluded. INTERVENTION: Raloxifene, 60 $\mathrm{mg}, 2$ tablets daily; or raloxifene, $60 \mathrm{mg}$, 1 tablet daily and 1 placebo tablet; or 2 placebo tablets. MAIN OUTCOME MEASURES: New cases of breast cancer, confirmed by histopathology. Transvaginal ultrasonography was used to assess the endometrial effects of raloxifene in 1781 women. Deep vein thrombosis or pulmonary embolism were determined by chart review. RESULTS: Thirteen cases of breast cancer were confirmed among the 5129 women assigned to raloxifene vs 27 among the 2576 women assigned to placebo (relative risk $[R R], 0.24 ; 95 \%$ confidence interval $[\mathrm{CI}], 0.13-0.44 ; \mathrm{P} .001)$. To prevent 1 case of breast cancer, 126 women would need to be treated. Raloxifene decreased the risk of estrogen receptor-positive breast cancer by $90 \%$ (RR, $0.10 ; 95 \%$ CI, $0.04-0.24)$, but not estrogen receptor-negative invasive breast cancer (RR, 0.88; 95\% CI, $0.26-3.0$ ). Raloxifene increased the risk of venous thromboembolic disease (RR, 3.1; 95\% CI, 1.5-6.2), but did not increase the risk of endometrial cancer ( $R R, 0.8 ; 95 \%$ CI, 0.2-2.7). CONCLUSION: Among postmenopausal women with osteoporosis, the risk of invasive breast cancer was decreased by $76 \%$ during 3 years of treatment with raloxifene.

Publication Types: 7

* Clinical trial

* Multicenter study

* Randomized controlled trial

PMID: 10376571, UI: 99303064

1) Otros formatos: por medio de estos botones podemos acceder desde esta página a otros formatos como el "Citation" o el "Medline Report". También se nos ofrece el botón al link de los "Related articles" o artículos relacionados.

2) Fuente bibliográfica y 3) Título: igual que en el formato mínimo. 4) Autores: a diferencia que en el formato mínimo, aquí se muestran todos los autores del artículo, de hecho cuando se realiza una búsqueda por autor, la misma se realiza en todos ellos y no sólo en el principal.

5) Dirección o filiación del primer autor: este campo es muy importante para solicitar copias de los artículos, obtener el lugar de trabajo de los autores y en los más recientes (como el caso de nuestro ejemplo) la dirección de correo electrónico del citado autor.

6) Abstract: de tenerlo, recuerde que solamente el $76 \%$ de las citas del Medline lo tienen, el abstract del artículo es mostrado en esta sección.

7) Tipo de publicación y 8) Número identificatorio de MEDLINE y PubMed: igual que en el formato mínimo.

\section{Citation report:}

Esta forma de presentación solamente agrega algunos campos al anterior, siguiendo nuestro ejemplo, hasta la referencia número $\mathbf{8}$ es lo mismo y desde allí se observaría una imagen como la que sigue: (reproducimos aquí parcialmente los términos Mesh de un artículo)

\section{MeSH Terms:}

*Animal

* Animal Husbandry

* Carcinogens/analysis*

* Cattle

* Drug Residues/analysis*

* Drug Residues/adverse effects

* Estradiol/analysis*

* Estradiol/adverse effects

* European Union

* Food Contamination*

* Human

* Meat/analysis*

* Meat/adverse effects

* Mutagens/analysis

* Neoplasms/chemically induced

* Sex Hormones/analysis*

* Sex Hormones/adverse effects

* United States

* United States Food and Drug

Administration 
9) Términos MeSH: esta es la característica que justifica este tipo de formato, anteriormente ya se comentó que son los términos MeSH.

En la referencia se observan los términos como:

Encabezamientos o Headings

Términos principales o Major Terms (son aquellos que poseen el *)

Subencabezamientos asociados o Subheadings

Le siguen un campo Substances, de tratarse de temas relacionados a substancias.

\section{Medline report:}

Es el formato tradicional de presentación de dos caracteres por título de campo del MEDLINE, con todos los campos que existen en la base de datos, con los cuales se indexan los artículos. Este formato sólo se usa para obtener citas en programas de manejo bibliográfico.

Tenga en cuenta este formato de presentación ya que será el que deba elegir para guardar las búsquedas y luego importarlas en el programa de manejo bibliográfico Gelit que se describirá al final de este tutorial de Medline, con la finalidad de potenciar el almacenamiento de sus búsquedas en un entorno de base de datos.

La siguiente imagen muestra parcialmente el formato de presentación del "Medline report", en donde observamos que cada campo comienza con dos caracteres que serán descriptos con detalle más adelante:

\section{NCBI PubMed}

\section{PubMed QUERY}

PubMed ?

Other Formats: Citation

Links: Related Articles

UI - 0

$A U$ - Chedid $A$

$A U$ - Ryan LM

$A U$ - Dayal $Y$

$A U$ - Wolf $B C$

$\mathrm{AU}$ - Falkson G

TI - Morphology and Other Prognostic Factors of Hepatocellular Carcinoma.

LA - ENG

PT - JOURNAL ARTICLE

DA - 19990625

DP - 1999 Jun

IS - 0003-9985

TA - Arch Pathol Lab Med

PG - 524-528

IP -6

VI -123

JC $-79 Z$

$A B$ - OBJECTIVE: Hepatocellular carcinoma is a malignancy found worldwide that has typically poor....

PATIENTS AND METHODS: Our prior clinical study described 432 patients, but sufficient tissue was available...

RESULTS: Of the 224 patients, $71 \%$ were male, $65 \%$ white, and $73 \%$ over the age of 45 years. Ninety-one percent ...

CONCLUSION: This investigation suggests that histologic subtype and clinical features may provide useful ...

$A D$ - Department of Pathology, Finch University of Health Sciences/The Chicago Medical School, North Chicago, Ill.

PMID- 0010383806

SO - Arch Pathol Lab Med 1999 Jun;123(6):524-528

\section{Limitando nuevamente la búsqueda:}

En la página de resultados existe la posibilidad de limitar nuevamente la recuperación de citas si así lo deseáramos.

Si se desea incrementar el número de citas mostradas por página (en un máximo de 5000), se debe seleccionar la cantidad que se desea del menú desplegable a la derecha de la leyenda "Docs Per Page:", luego oprimir "Search", con lo cual se realizará la misma búsqueda mostrando las citas con la cantidad por página ahora seleccionada.

Si lo que se desea es acotar el número de citas a un período determinado (opción ya ofrecida en la página principal), se debe desplegar el menú de "Date limit" y seleccionar el rango, luego oprimir "Search" nuevamente y PubMed nos mostrará las citas dentro del período de tiempo seleccionado.

NCBI PubMed PubMed QUERY PubMed ?

(past 1 Year only)

$\begin{array}{lll}\text { Details } & \text { neoplasm } & \text { Search Clear } \\ & 10-\text { Entrez Date Limit: } 1 \text { Year }-\end{array}$

Docs Per Page: 10 Entrez Date Limit: 1 Year $\checkmark$

citations 1-10 displayed (out of 51054 found) page 1 of 5106

Display Medline report - for the article selected (default all)

Si la cantidad de citas encontradas supera el límite de las mostradas por página, aparece un botón al final de la página ("Go to") que nos posibilita ir a la siguiente (o a otra que seleccionemos) para ver mas citas, recordar que si marcamos artículos con el botón de selección, estos se tienen en cuenta para cuando se pida mostrar todas las citas elegidas.

\section{$\square$ Chedid A. et al [See Related Articles]}

Morphology and Other Prognostic Factors of Hepatocellular Carcinoma. Arch Pathol Lab Med. 1999 Jun;123(6):524-528.

[Record as supplied by publisher] PMID: 10383806.

Order documents on this page through Loansome Doc

$$
\text { This is page } 1 \text { of } 5106
$$$$
\text { Go To Page } 2 \text { - }
$$

\section{Enlace a artículos "full-text":}

Una vez seleccionados los artículos para ver (recordar que si no se elige ninguno, por defecto se muestran todos), y elegido el formato en el cual se va a ver, al oprimir el botón "Display" se nos muestra la página de resultados con los artículos y el formato seleccionado. En esta página (recordar que ahora ya no es posible modificar el número de citas por página ni el período de tiempo, si desea hacerlo debe oprimir el boton "back" o "atrás" del navegador) algunas citas de PubMed tienen links a sitios web de editores de journals, en el cual se encuentra disponible la versión de texto completo o "full-text" de dichos artículos.

Para estas citas aparecerá un botón con el nombre del journal (p.e. 


\section{NCBI PubMed \\ Other Formats: MEDLINE \\ Links: Related Articles free BMJ \\ $\square$ Order this document \\ BMJ 1998 Jun 27:316(7149):1959-61}

PubMed QUERY

PubMed ?

\section{Evaluating information technology in health care: barriers and challenges.}

Heathfield H, Pitty D, Hanka R

J. Nutr) o el genérico de "Journal Button" , también podría aparecer un botón con el mensaje de "Get Original Text" .

Por ejemplo si encontramos un artículo que nos interesa que está en el British Medical Journal, en los botones de Links, a la derecha de Related Articles aparece (como se muestra en la imagen) el botón con el link al sitio del BMJ con la posibilidad de ver el artículo completo en full-text:

Siguiendo el link en este caso veríamos el artículo en full-text como lo muestra la siguiente imagen:

Para participar, los editores de journals, deben enviar la versión elec-

\section{BMJ}

Home Help Search/Archive Feedback Table of Contents

BMJ 1998; 316: 1959-1961 (27 June)

Information in practice

- Send a response to this article - Electronic responses to this article

Evaluating information technology in Search Medline for articles by: health care: barriers and challenges.

Heathfield, H. Hanka, R.

Alert me when:

New articles cite this article tra disponible para consulta. Esta característica, si bien comenzó siendo un proyecto experimental se convierte en uno de los atractivos más interesantes de PubMed, se espera que la lista de Journals suscriptos a este sistema crezca día a día.

\section{Artículos relacionados:}

Otra de las características diferenciales de PubMed es la capacidad de encontrar documentos que son similares a un documento que usted encontró de su interés.

PubMed compara las palabras del título y del abstract tanto como los términos MeSH de cada cita con un poderoso algoritmo de comparación de palabras. Por medio de éste, selecciona los artículos que coinciden mejor con el evaluado. El mejor resultado es guardado y almacenado en en un set precalculado. El botón de "Related Articles" trae en orden de relevancia y en cronología decreciente los artículos relacionados.

Si usted encontró uno o más atrículos que describen exactamente lo que busca, usando esta función incrementará la especificidad de su búsqueda en relación a las vías convencionales.

\section{Guardando o imprimiendo una búsqueda}

Por último, al final de la página aparece el botón de guardado ("Sa$v e ")$, con opciones para Macintosh, PC o Unix, y el formato deseado, Text o HTML. Luego de oprimir el botón "Save" y elegir el sistema y el formato, el navegador nos preguntará en donde guardar dicho archivo.

El botón "Order" sirve para pedir los artículos seleccionados por

\section{Save the above report in $\begin{array}{llll}\text { Macintosh } \nabla & \text { Text } \quad \nabla \\ \text { Format: }\end{array}$ \\ Order documents on this page through Loansome Doc}

intermedio de "Loansome Doc", un sistema para la obtención de los artículos impresos, mediante una red americana de bibliotecas médicas.

Otra posibilidad para guardar o imprimir una búsqueda, en este punto es ir al menú del navegador y en la opción "File" o "Archivo" seleccionar "Print" o "Imprimir" o "Save page as", o "Guardar como". De esta manera se puede guardar la búsqueda (se elige también el formato) o se la imprime directamente. termina puede requenir una subscripción o password, lo cual es de(http://www.ncbi.nlm.nih.gov/PubMed/fulltext.html) se encuen- 